Running Head: Distracted by pleasure

\title{
Distracted by Pleasure: Effects of Positive Versus Negative Valence on Emotional Capture Under Load
}

\author{
Rashmi Gupta, Young-Jin Hur, and Nilli Lavie \\ Institute of Cognitive Neuroscience, University College London, UK
}

\section{Corresponding Author}

Dr. Rashmi Gupta

Swiss Center for Affective Sciences

Campus Biotech

9, Chemin des Mines

CH-1202 Geneva, Switzerland

Tel +41-223790962

Email: rash_cogsci@yahoo.com 


\begin{abstract}
We report three experiments examining the effects of positive versus negative valence and perceptual load in determining attention capture by irrelevant emotional distractors. Participants performed a letter search task searching for one of two target letters $(\mathrm{X}$ or $\mathrm{N})$ in conditions of either low perceptual load (circular non-target letters) or high perceptual load (angular non-target letters that are similar to the target letters). On 25\% of the trials an irrelevant emotional distractor was presented at the display center and participants were instructed to ignore it. The distractor stimulus was either positive or negative and was selected from three different classes: IAPS pictures of erotica or mutilated bodies (Experiment 1), happy or angry faces (Experiment 2) and faces associated with gain or loss in a prior value-learning phase involving a betting game (Experiment 3 ). The results showed a consistent pattern of interaction of load and valence across the three experiments. Irrelevant emotional distractors produced interference effects on search RT in conditions of low load, with no difference between negative and positive valence. High perceptual load however consistently reduced interference from the negative-valence distractors, but had no effect on the positive-valence distractors. As these results were consistently found across three different categories of emotional distractors, they suggest the general conclusion that attentional capture by irrelevant emotional distractors depends on both their valence and the level of perceptual load in the task and highlight the special status of distractors associated with pleasure.
\end{abstract}

Keywords: perceptual load, attentional capture, positive valence, negative valence, emotional distractors 


\section{Distracted by Pleasure: Effects of Positive Versus Negative Valence on Emotional}

\section{Capture Under Load}

The emotional value of information, as an evolutionary and heuristic device for understanding approach and avoidance signals, can have important sociobiological indications. In line with the significance of such indications, much research has emphasized the rapid detection of emotional information in a number of cognitive faculties such as perception (e.g. reduced visibility, see Nasrallah, Carmel \& Lavie, 2009) and attention (e.g. in attentional blink paradigms, McHugo, Olatunji \& Zald, 2013). Research also demonstrated that emotional information can capture attention, even when it is irrelevant to the task, distracting task performance (e.g. Hodsoll, Viding \& Lavie, 2012; Yiend, 2010 for review).

In contrast, a growing body of studies suggests that the perception of emotional information and its ability to capture attention may not always survive stronger tests of inattention to distractors. A strong test of inattention requires not only that the emotional information is entirely irrelevant to the task but also that the attended task involves a high level of perceptual load. This is because high perceptual load is necessary for engaging full attention capacity in the relevant task and thus guaranteeing complete inattention to the distractors (e.g. Lavie, 1995; Lavie \& Tsal, 1994). In support of this claim much research has shown that high perceptual load in the task (e.g. large search set size; complex perceptual discrimination) can eliminate distractor processing; while tasks of low perceptual load (small search set size; simple perceptual detection) result in a "spill over" of attention to the processing of irrelevant distractors (e.g. Lavie, 1995; Lavie, Hirst, De Fockert, \& Viding, 2004; see Lavie, 2005; 2010 for reviews). It is important to note that the effects of perceptual load generalize to distractors that are entirely irrelevant to the task at hand (e.g. Forster \& Lavie, 2008a;b), because emotional distractors may also be irrelevant to the attended tasks (for example angry faces distractors presented during a task of letter search). 
Indeed there is now evidence to suggest that attention capture by emotional distractors critically depends on the level of perceptual load in the task. However, as we briefly review below, this evidence is typically based on assessing the processing of negative emotional stimuli (e.g. fearful faces as compared to faces with a neutral expression). The impact of distractor valence (i.e. whether it conveys a negative or positive emotional information) on attentional capture, and the potential interaction of valence and load have not as yet been addressed.

This was the aim of the present research. We report a comprehensive study of the interactive effects of emotional valence and task load for three classes of emotional information: emotional scenes, face expressions, and learned emotional valence (associated with either gain or loss in a choice task). Before we describe our method we shall briefly review the relevant previous research.

One of the first tests of the role of perceptual load in processing emotion did include both negative and positive information (fearful and happy faces, vs. neutral faces), and demonstrated that the differential amygdala signal related to the different irrelevant emotional faces was eliminated in conditions of high perceptual load of the task (involving subtle orientation discrimination (Pessoa et al., 2002). However, the baseline response to both emotional expressions in conditions of low load was not assessed in this study. Instead, the high load task condition was compared to a condition of full attention to the emotional stimuli. Therefore it remains unclear whether there would be an effect of valence on amygdala response (and presumably associated capture of attention) for these emotional distractors had they been presented as irrelevant distractors in conditions of low load. In other words, while negative and positive stimuli may not differ when tested at ceiling (full attention condition) or at floor (high load conditions) they may still differ in the range of attention availability between ceiling and floor levels. 
While all the follow-up studies have included low load conditions, these tests were limited to using negative emotional distractors only. Pessoa, Padmala, and Morland (2005) compared the amygdala response to negative (vs. neutral) fearful faces presented as irrelevant stimuli during performance of a line orientation discrimination task. Perceptual load of the orientation discrimination task was varied by changing the similarity of the orientations used. The results indicated that the emotional irrelevant faces elicited an amygdala response under conditions of low perceptual load, suggesting that emotional faces captured attention, despite their task irrelevance. However, this effect was eliminated under increased levels of perceptual load. These findings extend across both foveal presentation (as in Pessoa and colleagues studies) and peripheral presentations of the fearful faces (Silvert et al, 2007) and apply to other load tasks (e.g. letter search, Bishop et al. 2007; Mitchel et al., 2007) and to both low and highly anxious people (Bishop et al.2007). In all cases the irrelevant negative (fearful) faces led to a differential amygdala response (compared to neutral face conditions) in conditions of low perceptual load in the attended task and this response was eliminated with higher perceptual load in the task. This line of studies suggests not only that negative emotional face expression requires attention to elicit an amygdala signal related to emotion perception, but also that negative face expressions cannot capture attention when attention is fully engaged in the task (in conditions of high perceptual load).

Face expressions are perhaps not as emotionally laden and arousing as some of the emotional scenes depicted in the International Affective Picture System (IAPS). It is plausible, for example, that images of mutilated bodies and those displaying erotic engagement would trigger a stronger emotional response and arousal than the sight of happy or angry faces. Would attention capture effects by the emotionally laden IAPS images survive strong tests of inattention and persist when these are irrelevant to attended tasks of high perceptual load? Two studies (Erthal et al., 2005; Okon-Singer, Tzelgov \& Henik, 2007) 
suggest that capture of attention by IAPS images of mutilated bodies is not immune to the effects of perceptual load. In both studies mutilated-body images were presented as irrelevant distractors while subjects performed another task (either involving orientation discrimination, or a letter search, (see Note 1)) with varying levels of perceptual load. Perceptual load was manipulated either through orientation similarity (as in the previous studies by Pessoa and colleagues) or search set size. The results showed that while the irrelevant negative images produced interference effects on the task RT in conditions of low load, interference effects were eliminated with higher perceptual load.

These findings provide further support for the conclusion reached by the emotional face studies that attention capture by emotional distractors does not survive strong tests of attention, that involve task conditions of high perceptual load. However, as we mention earlier the emotional distractors used were of a negative valence. This leaves open the possibility that a different pattern would emerge for positive emotional stimuli.

In the present study we thus set out to establish the differential effects of valence (either positive or negative) on attentional capture under different levels of perceptual load in the task (varied by the similarity of the target and non-target letters in a visual search task, see Lavie \& Cox, 1997). To achieve full and general understanding of the interactive effects of emotional valence and load we examined this for emotional expressions of distractor faces; emotional scenes (negative images of mutilated bodies or positive images of eroticism); as well as for stimuli of learned emotional values (based on learning their associations with monetary gain or loss).

Note that in the latter case the very same faces are associated with either negative or positive emotion and thus clearly allows us to address the effects of emotional value of information, while controlling for any visual differences (e.g. greater contrast for some face expression with an open mouth versus closed mouth expression). 
By using these three different categories of emotional stimuli, any consistent difference between attentional capture by negative as compared to positive stimuli is likely to reveal a general effect of valence, rather than stimulus-specific effects. Moreover, the direct comparison between negative and positive emotional distractors provides the opportunity to match the level of reported arousal between negative and positive valence and thus rule out arousal-related accounts.

In addition, to design a robust measure of attentional capture by the irrelevant emotional distractors we followed Forster and Lavie's (2008) method of presenting the distractors with low probability (e.g. on only $25 \%$ of the trials, with distractor absent on the rest of the trials, Note 2). This should allow us to establish an attentional capture measure that is not moderated by habituation (see Forster \& Lavie, 2008a). Finally since two of our experiments involved faces and face processing is known to be degraded in the periphery, we presented all distractor stimuli in the display centre, directly at the position of eye fixation.

\section{Experiment 1}

\section{Method}

Participants. All the experiments reported were approved by the departmental ethics committee of the Institute of Cognitive Neuroscience at University College London.

In exchange for either course credit or money a total of 14 right-handed participants reporting normal or corrected-to-normal vision participated in this experiment, after giving informed consent. One participant was removed from the analysis due to high error rate $(\mathrm{M}=$ $34 \%$ ) in the letter search task. The remaining participants (3 males) had an age range of 19-31 years $(M=22$ years, $S D=3$ years $)$.

Stimuli \& Procedure

Stimuli were displayed on a 15-inch color monitor $(75 \mathrm{~Hz}, 32$-bit true colour; resolution $1024 \times 768$ pixels) viewed from a distance of $60 \mathrm{~cm}$, held fixed with a chin-rest. E- 
Prime 1.1 software (Schneider, Eschman, \& Zuccolotto, 2002) operating on a computer with a $3.4 \mathrm{GHz}$ Pentium 4 processor generated stimuli and recorded responses acquired via a number pad.

Each trial began with a centrally presented fixation point for $500 \mathrm{~ms}$, followed immediately by a $500 \mathrm{~ms}$ presentation of the stimulus display. The stimulus display consisted of a distractor image subtending $12^{\circ}$ horizontally by $6.6^{\circ}$ vertically at the centre of the screen, with two letter strings of three letters each appearing in a horizontal row above and below the image. Two classes of images were employed: positive and negative. Positive images consisted of erotic photographs, and negative images consisted of photographs of mutilated bodies. Ninety-six pictures (48 pleasant and 48 unpleasant) were randomly selected from the International Affective Picture System (IAPS) (Lang, Bradley, \& Cuthbert, 2005). Following the protocol developed by Lang and colleagues, all images were assessed on a 1-9 scale in terms of valence (from positive to negative) and arousal (from low to high) prior to the experiment by 7 participants ( 3 males) of a similar age $(M=25$ years, $S D=5$ years $)$ to that of the experiment participants. Forty-eight pictures were selected for the database based on the participant ratings and of these twenty-four pictures (12 positive and 12 negative) with matching arousal ratings $(\mathrm{M}$ arousal $=7.12$ for the pleasant images and $\mathrm{M}$ valence $=2.73 ; \mathrm{M}$ arousal $=7.40$ for the negative images and $\mathrm{M}$ valence $=8.49$ ) were randomly selected for the Experiment. There was a significant difference in valence rating between positive and negative pictures, $t(1,11)=34.8, p<.001$. Both positive and negative pictures were arousal matched, $t(1,11)=1.32, p=.21$

The background color was black. The letters were presented in grey color (hue, saturation, light: $0,0 \%, 75 \%)$. One target letter $(\mathrm{X}$ or $\mathrm{N})$ and five non-target letters (' $\mathrm{O}$ ' in the low-load and H', 'K', 'W', 'M', 'Z' on the high-load) were presented in a random order in 
each trial. Each letter measured $0.85^{\circ} \times 0.91^{\circ}$ and the distance between each letter (measured from letter centre to centre) in the horizontal row was $6.9^{\circ}$.

Participants were required to search the letter string for a target letter (either $\mathrm{X}$ or $\mathrm{N}$ ) and made a speeded response using the numerical keypad by pressing the 0 key if the target was an $\mathrm{X}$ and the 2 key if the target was an N. Participants were instructed to ignore the distractor image. A tone gave feedback for errors or failures to respond within $2 \mathrm{sec}$. The response time window was followed by an inter trial interval of $1900 \mathrm{msec}$.

There were one low-load and one high-load block of 48 trials in each block. Each block contains $75 \%$ distractor absent trials (in which no distractor was presented) and $25 \%$ distractor present trials ( $12.5 \%$ for each valence category: positive and negative). The block orders, target positions and distractor conditions were counterbalanced across all participants before any exclusion. The order of trials within each block was random.

\section{Insert Figure 1 about here}

\section{Results and Discussion}

\section{Reaction Times and Errors}

Mean RT and error rates were entered into within-subject ANOVAs with the factors of perceptual load (low, high) and distractor condition (pleasant distractor, unpleasant distractor, distractor absent).

RT. The RT ANOVA revealed a significant main effect of load, $F(1,12)=63.2, M S E$ $=40596.3, p<.001, \eta_{\mathrm{p}}{ }^{2}=0.841$. Mean RT was higher in the high-load $(\mathrm{M}=992 \mathrm{~ms})$ than in the low-load $(\mathrm{M}=656 \mathrm{~ms})$ condition, confirming that our perceptual-load manipulation was effective. There was a significant main effect of distractor condition, $F(2,24)=11.8, M S E=$ 7769.0, $p<.001, \eta_{\mathrm{p}}{ }^{2}=0.454$. As can be seen in Table 1 , compared to the distractor absent condition ( $\mathrm{M}=728 \mathrm{~ms}$ ) search RT was significantly longer in the presence of both the 
positive $(\mathrm{M}=845 \mathrm{~ms}), t(12)=6.58, p<.001$, and negative distractors $(\mathrm{M}=803 \mathrm{~ms}), t(12)=$ 3.04, $p<.01$. There was no significant difference in RTs between positive and negative distractors, $t(12)=1.44, p=.17$. Importantly, there was also a significant interaction between load and distractor condition, $F(2,24)=9.96, M S E=3928.7, p<0.01, \eta_{\mathrm{p}}{ }^{2}=0.472$. As seen in Table 1, in the low-load condition RT was slower in the presence (vs. absence) of both positive, $t(12)=5.73, \mathrm{p}<.001$, and negative distractors, $t(12)=4.83, p<.001$, with no significant difference in RTs between positive and negative distractors $(t<1)$. In the highload condition, RT did not differ between the negative distractor and the no-distractor conditions, $t(12)=.116, p=.910$, however, RT was significantly longer in the presence of positive distractors as compared to both the no-distractor condition, $t(12)=5.45, p<.001$ and the negative distractor condition, $t(12)=2.42, p<.05$.

Insert Table 1 about here

An additional analysis on the calculated scores of distractor interference effects (RT in the presence minus absence of each distractor type, namely negative distractor RT minus distractor absent RT, and positive distractor RT minus distractor absent RT) confirmed this pattern. Load significantly interacted with valence, $F(1,10)=10.6, M S E=4738.33, p<.01$, $\eta_{\mathrm{p}}{ }^{2}=0.469$ so that distractor interference effects produced by the negative distractors were significantly reduced by high load, $t(1,12)=3.47, p<.01$, but distractor interference effects produced by the positive distractors were unaffected by load, $t(1,12)=.810, p=.43$ (Figure 2; see also Note 3). 
Error rates. Error rates included trials in which no response was made within the $2 \mathrm{~s}$ time window. (These constituted less than $1 \%$ in all the experiments reported in this paper). The ANOVA on the error rates revealed a significant main effect of load, $F(1,12)=27.7$, $M S E=0.021, p<.001, \eta_{\mathrm{p}}{ }^{2}=0.698$. Error rate was higher in the high-load $(\mathrm{M}=18 \%)$ than in the low-load $(\mathrm{M}=10 \%)$ condition, further confirming that the perceptual-load manipulation was effective. There was also a significant main effect of distractor condition, $F(2,24)=$ 3.97, MSE $=0.022, p<.05, \eta_{\mathrm{p}}^{2}=0.249$. Error rate in letter-search task was significantly higher in the presence of positive $(\mathrm{M}=21 \%), t(12)=2.12, p<.001$, and negative distractors $(\mathrm{M}=23 \%), t(12)=3.91, p<.01$ compared to distractor-absent condition $(\mathrm{M}=12 \%)$. There was no significant difference in error rate between positive and negative distractors, $t(12)$ $=.262, p=.79$. These results replicate the $\mathrm{RT}$ results. There was no significant interaction between load and distractor condition, $F(2,24)=.183, M S E=0.029, p=.83, \eta_{\mathrm{p}}{ }^{2}=0.015$.

Overall the results of the Experiment 1 demonstrate that high perceptual load eliminates distractor interference from distractors of negative valence, but has no effect on interference effects of positive-valence distractors. The modulation of capture by negative IAPS distractors is consistent with previous reports (e.g. Erthal et al., 2005; Okon-Singer et al., 2007), however the findings that positive IAPS distractors continue to capture attention under high load is novel. It is important to note that since the positive distractors were matched on arousal to the negative distractors in this study the differential effect of load on capture effects by the distractors of different valence cannot be attributed to difference in arousal. Indeed as Figure 2 shows the interference effects of the positive and negative distractors were of similar magnitude in the conditions of low load. However, alternative accounts in terms of factors other than arousal remain. For example, the visual image properties differed between the negative and positive IAPS pictures. For example, there was less full figure or full figure parts information in the negative compared to the positive 
images due to their depiction of body parts and inclusion of splashes of blood over some of the parts as well. This could hinder perception of the negative image and thus reduce their ability to capture attention in the high load conditions, while the erotica content of the naked bodies or relevant body parts could be more easily perceived even during high load. Secondly, the specific content of erotica may be more attention capturing than the specific content of mutilated body parts (for example due to the triggering of a wider range of semantic associations).

Thus in Experiment 2 we aimed to better understand the role of emotional valence rather than visual and content differences between the stimulus classes, by examining the effects of perceptual load on distractor interference effects for faces that are visually similar but vary in emotional expressions (happy vs. angry).

\section{Experiment 2}

\section{Method}

\section{Participants}

In exchange for either course credit or money a total of 22 right-handed participants, reporting normal or corrected-to-normal vision, participated after giving informed consent. One participant was removed from the analysis due to high error rate $(\mathrm{M}=32 \%)$ in the letter search task and the remaining 21 participants (8 males) had an age range of 19-32 years $(\mathrm{M}=$ 22 years, $\mathrm{SD}=4$ years)

\section{Stimuli and Procedure}

The stimuli and procedure was similar to Experiment 1 except for the following changes. 12 faces of young adults (three males, three females), six for each happy or angry expression, all open-mouthed, were selected from the Karolinska Directed Emotional Face database (Lundqvist, Flykt, \& Ohman, 1998). Grayscale pictures of the faces subtending $12.3^{\circ} \times 9.5^{\circ}$ were presented in the center of the display as distractors on $25 \%$ of the trials, 
(12.5\% angry expression, $12.5 \%$ happy expression). The task letters subtended $0.95^{\circ} \times$ $0.76^{\circ}$ and were presented in a circular shape with a $6.2^{\circ}$ radius centred at fixation. All the other aspects of task stimuli and procedure were the same as in Experiment 1.

\section{Results and Discussion}

\section{Reaction Times and Errors}

Mean RT and error rates were entered into within-subject ANOVAs with the factors of perceptual load (low, high) and distractor condition (positive, negative, distractor absent).

RT. The ANOVA revealed a significant main effect of load, $F(1,20)=176.9, M S E=$ 26095.8, $p<.001, \eta_{\mathrm{p}}{ }^{2}=0.898$. Reaction time was higher in the high-load $(\mathrm{M}=967 \mathrm{~ms})$ than in the low-load ( $\mathrm{M}=599 \mathrm{~ms})$ condition, confirming again that our perceptual-load manipulation was effective. There was a significant main effect of distractor condition, $F(2$, $40)=12.4, M S E=4147.3, p<.001, \eta_{\mathrm{p}}{ }^{2}=0.384$. Compared to the no distractor condition $(\mathrm{M}$ $=727 \mathrm{~ms})$, search RTs was significantly higher in the presence of both the positive $(\mathrm{M}=796$ $\mathrm{ms}), t(20)=6.18, p<.001$, and negative face distractors $(\mathrm{M}=770 \mathrm{~ms}), t(20)=3.90, p<.001$. There was no significant difference in RTs between positive and negative face distractors, $t(20)=1.39, p=.18$. Importantly, there was also a significant interaction between load and distractor condition, $F(2,40)=4.66, M S E=5926.6, p<0.01, \eta_{\mathrm{p}}{ }^{2}=0.189$. As can be seen in Table 2, in the low-load condition RTs was significantly higher in comparison to the absent condition, in the presence of the positive, $t(20)=7.27, p<.001$, and negative face distractors, $t(20)=6.19, p<.001$. There was no significant difference in RTs between positive and negative face distractors in the low load, $t(20)=1.00, p=.328$. In the high-load condition, there was no significant difference in RT between the negative face distractor and the nodistractor conditions, $t(20)=.021, p=.984$, however, RT was significantly higher in the presence of positive face distractors as compared to both the no-distractor condition, $t(20)=$ $3.02, p<.01$ and the negative face distractor condition, $t(20)=2.07, p<.05$. 
Insert Table 2 about here

An ANOVA on the distractor interference effects (each conditions subtracted from the distractor absent condition as before) confirmed a significant interaction of load and distractor conditions, $F(1,20)=4.81, M S E=8707.81, p<.05, \eta_{\mathrm{p}}{ }^{2}=0.194$. As can be seen in Figure 3 load significantly reduced distractor interference effects from the negative distractor faces, $t(1,20)=2.82, p<.01$, but had no effect on interference by the positive distractor faces, $t(1,20)=.044, p=.96$.

Insert Figure 3 about here

Error rates. The error rate ANOVA revealed a significant main effect of load, $F(1,20)$ $=35.5, M S E=0.023, p<.001, \eta_{\mathrm{p}}{ }^{2}=0.640$. Error rate was higher in the high-load $(\mathrm{M}=21 \%)$ than in the low-load $(\mathrm{M}=5 \%)$ condition, which further confirm that the perceptual-load manipulation was effective. There was no significant main effect of distractor condition, $F(2$, $40)=.757, M S E=.011, p=.47, \eta_{\mathrm{p}}{ }^{2}=0.036$. There was also no significant interaction effect between load and distractor condition, $F(2,40)=.664, M S E=.012, p=.52, \eta_{\mathrm{p}}^{2}=0.032$.

Overall then Experiment 2 revealed the same pattern of results as that found in Experiment 1. Perceptual load eliminated distractor interference effects by the negative angry faces but had no effect on the interference effects from the positive happy faces. Once again the interference effects were similar in magnitude between the distractors of negative and positive valence in the conditions of low load, which suggests the interaction of load and valence is not due to a different baseline level of interference for the positive and negative valence. 
However although the angry and happy faces were more visually similar compared to the negative and positive IAPS images used in Experiment 1 (e.g. both were full faces and both open-mouthed) they still differed on some features, for example the shape of the eyebrows (see Figure 1B), which is known to be easily detected (e.g. Lundqvist, Esteves, \& Ohman, 1999).

We note that it is hard to explain the advantage for the happy over angry faces in terms of this difference since the pronounced eyebrow feature characterised the angry faces, (Figure 1). Nevertheless it was desirable to test if the same pattern of results would emerge with stimuli that do not differ at all in the negative and positive valence conditions. In Experiment 3 we therefore compared the effects of learned positive (gain in a betting game) or negative (a betting loss) value for the very same faces that were always with a neutral expression under each learned value. In addition the first two experiments involved short low load or high load blocks of trials and very few distractor presentations (to minimize distractor habituation). This however confines our conclusions to effects that are potentially only short-lived. In Experiment 3 we doubled the number of trials compared to the first two experiments, and this allowed us to examine whether the effects of valence on capture can be last over a longer period.

\section{Experiment 3}

Experiment 3 aimed to assess the effects of emotional valence on distractor processing for distractor stimuli that are the very same images in the negative and positive valence conditions. To that purpose participants engaged in a value learning procedure phase prior to the experiment, whereby neutral faces were associated with either loss or gain in a betting game. Value learning (VL) procedures are known to lead to the acquisition of strong positive or negative emotional responses towards the value learned stimuli (e.g. those associated with a monetary gain compared to those associated with a monetary loss). Brain 
imaging studies demonstrate that exposure to stimuli which have undergone VL activates the amygdala and anterior cingulate cortex, two regions of the brain commonly associated with response to first-order emotional items (see Fujiwara et al., 2009; Murray, 2007; Wiech \& Tracey, 2013, for a review). The question of interest in Experiment 3 was whether the effects of valence and load established in the first two experiments would be replicated for distractor stimuli that have acquired positive or negative emotional value following value learning at the start of the experiment.

\section{Method}

\section{Participants}

In exchange for either course credit or money 18 right-handed participants reporting normal or corrected-to-normal vision participated after giving informed consent. One participant was removed from the analysis due to high error rate $(M=33 \%)$ in the letter search task and the remaining 17 participants (11 males) had an age range of 19-35 years $(\mathrm{M}=$ 25 years, $\mathrm{SD}=4$ years).

\section{Stimuli \& Procedure}

Six upright static grayscale faces of young male adults bearing neutral expression were selected from Karolinska Directed Emotional Face database (Lundqvist, Flykt, \& Ohman, 1998) and arranged into three pairs, each pair appearing on one display. The study started with a VL task followed by the attention task.

VL Task: A pair of faces was presented on each trial (each subtending $12.3^{\circ} \times 9.5^{\circ}$ ), one face was presented $0.95^{\circ}$ above fixation and the other face was presented $0.95^{\circ}$ below fixation. Participants were informed that they are participating in a betting game and instructed to choose the face that would maximize point earnings in this game. Participant indicated their choice by pressing either the ' $\mathrm{T}$ ' key to indicate the top face in the pair, or the 'B' key to indicate the bottom face. Each display was presented until the participant made a 
response. Upon the participant response, a message indicating the outcome of the choice was presented in the center of the screen: either the word 'GAIN' in green, along with a "bing" sound; or the word 'LOSS' in red, along with 'bong" sound, or the word 'NOTHING' (no outcome) in yellow and no sound. Gains and losses were always worth 10 points (added or subtracted, respectively). A running total of points earned also appeared on the same display underneath the outcome word. Each face in the set was always paired with the same other face but their location (top or bottom) was randomized across trials. One face pairs always produced gains; one pair always produced losses; and one always produced no outcome (serving as the neutral condition). In each of the outcome (gain or loss) pairs one face predicted the outcome with high validity ( $80 \%$ probability, $20 \%$ probability of no outcome) and the other predicted an outcome of the same valence but with a low validity (20\% probability of outcome, $80 \%$ no outcome). There was one face pair for each category (gain, loss, or no outcome). Each pair was presented 100 times in randomly order, in a self-paced manner. To eliminate image effects, assignment of face pair categories and probabilities was counterbalanced across participants.

Letter-Search Task: The stimuli and procedure were the same as in Experiment 2 except for the following changes. The stimulus display was presented for $200 \mathrm{msec}$. There were one low-load and one high-load block of 96 trials in each block. Each block contained $75 \%$ distractor absent trials and $25 \%$ distractor present trials (12.5\% for each valence category: positive and negative). The order of block, target positions and distractor conditions were counterbalanced across participants.

\section{Results and Discussion}

\section{Learning Phase}

Following previous VL research (Raymond \& O’Brien, 2009) we adopted an exclusion criterion of minimum $65 \%$ correct choices of the loss and gain pairs on the last 30 
trials in the value-learning task. No subject had to be excluded following this criterion. There was no significant difference in learning performance between gain and loss pair, $t(1,16)=$ $1.65, p=.11$. For gain pairs, the high-probability win face $(\mathrm{EV}=0.8 \mathrm{x})$ was chosen on average on $90 \%(\mathrm{SD}=12 \%)$ of trials; for loss pairs, the low-probability loss face $(\mathrm{EV}=-$ $0.2 \mathrm{x})$ was chosen on $84 \%(\mathrm{SD}=14 \%)$ of trials; and for no-outcome control pair $(\mathrm{EV}=0)$, an arbitrarily selected face in each pair was chosen on $49 \%$ of trials $(\mathrm{SD}=15 \%)$.

\section{Letter-Search Task}

\section{Reaction Times and Errors}

Mean RT and error rates were entered into within-subject ANOVAs with the factors of perceptual load (low, high) and distractor condition (positive, negative, distractor absent).

RT. The ANOVA revealed a significant main effect of load, $F(1,16)=38.1, M S E=$ $10572.5, p<.001, \eta_{\mathrm{p}}^{2}=0.705$. Reaction time was higher in the high-load $(\mathrm{M}=671 \mathrm{~ms})$ than in the low-load $(\mathrm{M}=545 \mathrm{~ms})$ condition, confirming that the perceptual-load manipulation was effective, as before. There was a significant main effect of distractor condition, $F(2,32)$ $=6.03, M S E=705.9, p<.01, \eta_{\mathrm{p}}^{2}=0.274$. Compared to distractor absent condition $(\mathrm{M}=596$ $\mathrm{ms})$, search RTs was significantly higher in the presence of both the positive (M=617 ms), $t(16)=4.51, p<.001$, and negative face distractors $(\mathrm{M}=610 \mathrm{~ms}), t(16)=2.66, p<.01$. There was no significant difference in RTs between positive and negative face distractors, $t(16)<1$. Importantly, there was also a significant interaction between load and distractor condition, $F(2,32)=4.50, M S E=380.9, p<0.01, \eta_{\mathrm{p}}^{2}=0.220$. As can be seen in Table 3 , in the low-load condition RTs was significantly higher in, comparison to the absent condition, the presence of both the positive, $t(16)=3.48, p<.01$, and negative face distractors, $t(16)=$ 5.74, $p<.001$ and there was no difference in RT between them, $t(16)<1$. In the high-load condition, there was no difference in RT between the negative face distractor and the nodistractor conditions, $t(16)<1$; however, RTs was significantly higher in the presence of 
positive face distractors as compared to the no-distractor condition, $t(16)=2.93, p<.01$ and marginal significant difference to the negative face distractor condition, $t(16)=1.91, p=.07$. Similar to Experiment 1 and 2, results of Experiment 3 further confirm that high perceptual load does not filter out distractors with positive emotion.

Insert Table 3 about here

An ANOVA on the distractor interference effects (each conditions subtracted from the distractor absent condition as before) confirmed a significant interaction of load and distractor conditions, $F(1,16)=4.15, M S E=471.81, p<.05, \eta_{\mathrm{p}}{ }^{2}=0.206$. As can be seen in Figure 4 load significantly reduced distractor interference effects from the negative distractor faces, $t(1,16)=3.32, p<.01$, but had no effect on interference by the positive distractor faces, $t(1,16)<1$.

Insert Figure 4 about here

Error rates. The error rate ANOVA revealed a significant main effect of load, $F(1,16)$ $=38.9, M S E=0.009, p<.001, \eta_{\mathrm{p}}{ }^{2}=0.709$. Error rate was higher in the high-load $(\mathrm{M}=16 \%)$ than in the low-load $(\mathrm{M}=4 \%)$ condition, which further confirm that the perceptual-load manipulation was effective. There was no significant main effect of distractor condition, $F(2$, $32)=.380, M S E=0.006, p=.68, \eta_{\mathrm{p}}^{2}=0.023$. There was also no significant interaction effect between load and distractor condition, $F(2,32)=.627, M S E=0.002, p=.54, \eta_{\mathrm{p}}{ }^{2}=0.038$.

Overall, Experiment 3 results replicated the pattern found in Experiments 1-2, with distractor stimuli that were all neutral faces and only differed in their learned positive or 
negative valence. It also demonstrated that the effects can be found over a longer period (with twice as many trials) compared to the first two experiments.

\section{General Discussion}

The present study established the effects of emotional valence and perceptual load on the extent to which entirely irrelevant distractors can capture attention and interfere with task performance. In three experiments involving images of mutilated body parts or erotic content (Experiment 1), emotional face expressions of either anger or happiness (Experiment 2), and neutral faces with a learned value of either loss or gain (Experiment 3), for negative and positive valence respectively, the same pattern of results emerged. The results consistently demonstrated an interactive effect of load and valence. In conditions of low load, distractors of both negative and positive valence captured attention and interfered with task performance, to a similar extent, despite their complete irrelevance to the letter search task. High perceptual load, however was found to reduce distractor interference effects for the distractors of negative valence, but did not affect distractors of positive valence. Positive distractors interfered with performance irrespective of the level of load in the task. As the results were generalized across different stimulus classes and different semantic emotional contents (i.e. erotic vs. gory; happy vs. angry, gain vs. loss) they highlight the general role of overall emotional valence, while ruling out alternative accounts in terms of different visual appearance or semantic content between negative and positive stimuli. The present results clearly suggest that the valence of emotional information needs to be considered together with the level of perceptual load in determining attentional capture.

\section{Relation to previous research:}

Negative valence. The findings the high perceptual load reduced distractor interference from negative distractors across the three categories of emotional content accord with previous findings that show that attention capture by both faces of negative expression 
(Bishop et al. 2007; Mitchel et al., 2007; Pessoa, Padmala, and Morland 2005; Silvert et al, 2007) and images of negative valence (Erthal et al., 2005; Okon-Singer, Tzelgov \& Henik, 2007) is reduced in conditions of high perceptual load in the task. The present study extends this pattern to a paradigm which provides a sensitive measure of attentional capture from low-probability distractors which results in robust distractor interference in the conditions of low perceptual load (compare for example the circa $25 \mathrm{msec}$ distractor interference effects in the low load condition of Okon-Singer et al., 2007 to the of $146 \mathrm{msec}$ effect found for the same negative image class in the present study (Experiment 1). Clearly high perceptual load is shown to be a strong modulator of attention capture by the negative distractors in our paradigm. Moreover our study extends the effects of perceptual load to the class of distractors of learned negative value, which do not differ in their visual appearance to the value-learned positive distractors.

Positive valence. The consistent findings that interference by positive distractors is unaffected by high perceptual load demonstrate an attention-capture advantage for positive stimuli, under demanding task conditions. The positive advantage in attention capture may at first sight appear somewhat surprising since it is at odds with the potential evolutionary benefit of being attuned to threat, as well as with previous findings of an advantage for negative over positive stimuli in processes of detection and search (e.g. Dijksterhuis \& Aarts, 2003; Nasrallah et al., 2009; Eastwood, Smilek, \& Merikle, 2001; Huang, Chang, \& Chen, 2011). However, it is plausible that while information of negative valence has a priority at early perceptual stages of detection leading to a detection advantage and facilitating search for negative stimuli; information of positive valence is better capable of attracting and capturing attention, even when people try to ignore it under conditions of high perceptual load. In other words, whereas early processes of detection and perception may favour 
negative stimuli, when it comes to suprathreshold stimuli presented as distractors, it may be harder to ignore those distractors that involve a positive valence.

This interpretation is consistent with a growing body of studies demonstrating an advantage for positive over negative emotional information in attracting and capturing attention even when the information is not directly relevant to the task. For example, following the presentation of emotional faces identification of target letters is facilitated in the location of the previously presented happy faces as compared to the sad faces, although the emotional face expression is clearly irrelevant to the letter identification (Srivastava \& Srinivasan, 2010)

The result is consistent with the happy emotion advantage found with schematic faces (Mack \& Rock, 1998; Becker \& Leinenger, 2011) as well as real faces (Gupta \& Srinivasan, 2014) using inattentional blindness (IB) paradigms. For example Mack \& Rock (1998) presented a smiley or a sad face, unexpectedly on the last trial, in the display periphery, while subjected performed a line length discrimination task concerning cross shapes presented at fixation. Inattentional blindness rates were lower for the smiley faces compared to the sad faces. Becker and Leinenger (2011) combined a mood induction procedure with assessment of inattentional blindness for faces with a positive or negative expression presented as irrelevant distractors while participants perform a motion tracking task. Participants tracked a selected set of moving discs while an unexpected face appeared on an irrelevant disc to those tracked. Participants were then asked whether they noticed anything unusual as is typical in inattentional blindness procedures. The findings revealed a mood congruency effects on inattentional blindness rates to the distractor faces, such that face expression of the same valence as that of the induced mood were more likely to be detected. Of most relevance to the present research are the findings that under the neutral mood conditions, rates of inattentional blindness were lower for happy faces compared to sad faces. Gupta and Srinivasan (2014) 
measured the effects of perceptual load in a letter search task (varied similarly to the present study) on the rates of recognition (measured with 2 alternative forced choices) of an unexpected picture of face appearing on the last trial of the task. Their results pattern is similar to the present findings. High perceptual load reduced recognition of the sad faces but had no effect on recognition of the happy faces. In the attentional blink paradigm positive erotic images were found to produce a greater decrement ("blink") to detection of following neutral stimuli compared to negative images (e.g. of fear or disgust) (Ciesielski et al., 2010, Most et al., 2007). The findings that faces associated with a gain (in a similar value-learning procedure through betting as that used here) appear immune to attentional blink themselves while faces associated with a loss do suffer from attentional blink (Raymond \& O'Brien, 2009) is also in support of an attentional capture advantage for stimuli of positive over negative valence, in conditions of high perceptual load (as is characteristic of the rapid visual presentation in the attentional blink paradigm.

Finally, negative emotional stimuli are sometimes found to captivate and "hold" attention for longer than positive stimuli (for example negative emotional stimuli are found harder to disengage when presented as an invalid cue, e.g. Fox et al., 2001; Horstmann, Scharlau, \& Ansorge, 2006) even in non-anxious people. Moreover this negative advantage in attention "holding" is found in cases when there is no valence effect on the component of attention capture (e.g. in orienting attention to the cued emotion) similarly to our low load results. We note that our paradigm could not differentiate between the effects of valence on capturing attention and those on the latency of holding and captivating attention. Both effects of capturing and captivating would simply show up as increased latency to respond to the search target in our task. Nevertheless, our findings that high load eliminates any effect of negative valence stimuli on RT clearly demonstrate that negative distractors are unable to either capture nor captivate attention when attention is fully engaged in the task. 
Implications for daily life and future research. Our findings that positive distractors can capture attention under conditions of high information load may have important implications for daily life. For example, when wishing to capture attention of another person it should be effective to approach them with a smile. When attempting to focus attention in a task one may want to eliminate any sources that are likely to convey pleasant emotional information and therefore distract from task focus (e.g. turn off facebook notifications). The present findings have also clear implications for marketing. Positive messages can capture consumer attention even under in conditions of high perceptual load. In contrast, in order to perceive a negative message, for example a health warning on cigarette packaging, the rest of the package should involve only low perceptual load. Future research examining whether our findings apply to other distractor stimuli (e.g. words of negative or positive valence) and contents (e.g. stimuli conveying biological threat such as snakes and spiders, or biological reward such as food) as well as to peripheral distractor presentations (recall that in the present study the distractors were presented at eye fixation) should prove useful for a comprehensive understanding of the interaction of emotional valence and load in attention capture. 


\section{References}

Becker, M. W., \& Leinenger, M. (2011). Attentional selection is biased toward moodcongruent Stimuli. Emotion, 11, 1248-1254.

Bishop, S. J., Jenkins, R., \& Lawrence, A. D. (2007). Neural processing of fearful faces: effects of anxiety are gated by perceptual capacity limitations. Cerebral Cortex, 17, 1595-1603.

Ciesielski, B. G., Armstrong, T., Zald, D. H., \& Olatunji, B. O. (2010). Emotion modulation of visual attention: categorical and temporal characteristics. PLOS ONE 5:e13860.

Dijksterhuis, A., \& Aarts, H. (2003). On wildebeests and humans: The preferential detection of negative stimuli. Psychological Science, 14, 14-18.

Eastwood, J. D., Smilek, D., \& Merikle, P. M. (2001). Differential attentional guidance by unattended faces expressing positive and negative emotion. Perception and Psychophysics, 63, 1004-1013.

Erthal, F. S., De Oliviera, L., Mocaiber, I., Pereira, M. G., Machado- Pinheiro, W., Volchan, E., et al. (2005). Load-dependent modulation of affective picture processing. Cognitive, Affective, \& Behavioral Neuroscience, 5, 388-395.

Forster, S. \& Lavie (2008a). Attentional capture by entirely irrelevant distractors. Visual Cognition, 16, 200-214.

Forster, S., \& Lavie, N. (2008b). Failures to ignore entirely irrelevant distractors: the role of load. Journal of Experimental Psychology: Applied, 14, 73.

Fox, E., Russo, R., Bowles, R., \& Dutton, K. (2001). Do threatening stimuli draw or hold visual attention in subclinical anxiety?. Journal of Experimental Psychology: General, 130(4), 681-700. 
Fujiwara, J., Tobler, P. N., Taira, M., Iijima, T., \& Tsutsui, Ken-Ichiro (2009). Gegregated and integrated coding of reward and punishment in the cingulate cortex. Journal of Neurophysiology, 101, 3284-3293.

Gupta, R., \& Srinivasan, N. (2014). Only irrelevant sad but not happy faces are inhibited under high perceptual load. Cognition and Emotion, 7, 1-8.

Hodsoll, S., Viding, E., \& Lavie, N. (2011). Attentional capture by irrelevant emotional distractor faces. Emotion, 11, 346-353.

Horstmann, G., Scharlau, I., \& Ansorge, U. (2006). More efficient rejection of happy than of angry face distractors in visual search. Psychonomic Bulletin \& Review, 13(6), 10671073.

Huang, S., Chang, Y., \& Chen, Y. (2011). Task-Irrelevant angry faces capture attention in visual search while modulated by resources. Emotion, 11, 544-552.

Lang, P. J., Bradley, M. M., \& Cuthbert, B. N. (2005). International affective picture system (IAPS): Affective ratings of pictures and instruction manual. NIMH, Center for the Study of Emotion \& Attention.

Lavie, N. (1995). Perceptual load as a necessary condition for selective attention. Journal of Experimental Psychology: Human Perception and Performance, 21, 451.

Lavie, N. (2005) Distracted and confused?: selective attention under load. Trends in Cognitive Sciences, 9, 75-82.

Lavie, N. (2010). Attention, Distraction and Cognitive Control under Load. Current Direction in Psychological Science, 19, 143-148.

Lavie, N. \& Cox, S. (1997). On the efficiency of attentional selection: Efficient visual search results in inefficient rejection of distraction. Psychological Science, 8, 395-398.

Lavie, N., Hirst, A., De Fockert, J. W. \& Viding, E. (2004) Load theory of selective 
attention and cognitive control. Journal of Experimental Psychology: General, 133, $339-354$.

Lavie, N. \& Tsal, Y. (1994). Perceptual load as a major determinant of the locus of selection in visual attention. Perception \& Psychophysics, 56, 183-197.

Lundqvist, D., Esteves, F., \& Öhman, A. (1999). The face of wrath: Critical features for conveying facial threat. Cognition and Emotion, 13, 691-711.

Lundqvist, D., Flykt, A., \& Öhman, A. (1998). The Karolinska Directed Emotional Faces KDEF, CD ROM from Department of Clinical Neuroscience, Psychology section, Karolinska Institute, ISBN 91-630-7164-9.

Mack, A., \& Rock, I. (1998). Inattentional blindness (pp. 286-290). Cambridge, MA: MIT press.

McHugo, M., Olatunji, B.O., Zald, D.H. (2013). The emotional attentional blink: what we know so far. Frontiers in Human Neuroscience, 7, 151.

Mitchell, D. G. V., Nakic, M., Fridberg, D., Kamel, N., Pine, D. S., \& Blair, R. J. R. (2007). The impact of processing load on emotion. Neuroimage, 34, 1299-1309.

Most, S. B., Smith, S. D., Cooter, A. B., Levy, B. N., \& Zald, D. H. (2007). The naked truth: positive, arousing distractors impair rapid target per- ception. Cognition \& Emotion, 21, $964-$ 981.

Murray, E. A. (2007). The amygdala, reward, and emotion. Trend in Cognitive Sciences, 11, 489-497.

Nasrallah,M., Carmel,D., Lavie,N. (2009). "Murder she wrote":Enhanced sensitivity to negative word valence. Emotion 9, 609-618.

Okon-Singer, H., Tzelgov, J., \& Henik, A. (2007). Distinguishing between automaticity and attention in the processing of emotionally significant stimuli. Emotion, 7, 147-157. 
Pessoa, L., McKenna, M., Gutierrez, E., \& Ungerleider, L. G. (2002). Neural processing of emotional faces requires attention. Proceedings of the National Academy of Sciences, 99, 11458-11463.

Pessoa, L., Padmala, S., \& Morland, T. (2005). Fate of the unattended fearful faces in the amygdala is determined by both attentional resources and cognitive modulation. NeuroImage, 28, 249-255.

Raymond, J. E., \& O’Brien, J. L. (2009). Selective visual attention and motivation: The consequence of value learning in an attentional blink task. Psychological Science, 20, 981-988.

Schneider, W., Eschman, A., \& Zuccolotto, A. (2002). E-Prime: User's guide. Psychology Software Incorporated.

Silvert, L., Lepsien, J., Fragopanagos, N., Goolsby, B., Kiss, M., Taylor, J. G., Raymond, J. E., Shapiro, K. L., Eimer, M., \& Nobre, A. C. (2007). Influence of attentional demands on the processing of emotional facial expressions in the amygdale. Neuroimage, 38, 357-366.

Srivastava, P., \& Srinivasan, N. (2010) Time course of visual attention with emotional faces. Attention, Perception, \& Psychophysics, 72, 369-377.

Wiech, K., \& Tracey, I. (2013). Pain, decisions, and actions: a motivational perspective. Frontiers in Neuroscience, 7, doi: 10.3389/fnins.2013.00046.

Yiend, J. (2010). The effects of emotion on attention: A review of attentional processing of emotional information. Cognition \& Emotion, 24, 3-47. 


\section{Acknowledgments}

This research was supported by a post-doctoral fellowship to Dr. Rashmi Gupta from the Marie Curie Incoming Research Fellowship. 


\section{Footnotes}

Note 1. Okkon-singer et al. (2007) presented also positive IAPS images of erotic nature however they did not report the results for this category.

Note 2. We note that the presence or absence of a distractor has altered the number of items in the display overall (display size $=\mathrm{n}$ in the distractor absent conditions; $\mathrm{n}+1$ in the distractor present conditions) under both conditions of perceptual load in the task. This of course was a constant factor across our manipulation of perceptual load in the task and as such inconsequential for both our study design (an orthogonal $2 \times 3$ factorial design) and analyses. We thank an anonymous reviewer for raising this point.

Note 3. To verify that our findings are not due to a small number of trials with extreme RT we also repeated the same ANOVAs on the median RTs (which are insensitive to extreme values). The results pattern and significance did not change with the median RT analyses, thus ruling out this potential concern. Note also that our main finding of an interaction between load and valence was of large power $(\eta p 2=0.469)$ provides further reassurance. 


\section{Table Captions}

Table 1: Experiment 1: Mean RTs (SE in parentheses) and Percentage Error Rates as a Function of Distractor Condition and Load.

Table 2: Experiment 2: Mean RTs (SE in parentheses) and Percentage Error Rates as a Function of Distractor Condition and Load.

Table 3: Experiment 3: Mean RTs (SE in parentheses) and Percentage Error Rates as a Function of Distractor Condition and Load. 
Table 1

Distractor

Condition

\begin{tabular}{llll}
\hline & No Distractor & Negative & Positive \\
\hline Low-Load & $520(12)$ & $666(36)$ & $646(29)$ \\
RT (ms) & & & \\
\hline \%Error & 4 & 13 & 14 \\
\hline High-Load & $936(48)$ & $940(64)$ & $1045(49)$ \\
RT (ms) & & & \\
\hline \% Error & 20 & 33 & 29 \\
\hline
\end{tabular}


Table 2

\section{Distractor}

Condition

\begin{tabular}{llll}
\hline & No Distractor & Negative & Positive \\
\hline Low-Load & $521(15)$ & $609(27)$ & $590(15)$ \\
RT (ms) & & & \\
\hline \%Error & 4 & 3 & $1003(38)$ \\
\hline High-Load & $933(31)$ & $932(27)$ & \\
RT (ms) & & & 22 \\
\hline \% Error & 8 & 22 & \\
\hline
\end{tabular}


Table 3

\section{Distractor}

\section{Condition}

\begin{tabular}{llll}
\hline & No Distractor & Negative & Positive \\
\hline Low-Load & $527(21)$ & $555(22)$ & $552(21)$ \\
RT (ms) & & & \\
\hline \%Error & 6 & 30 & 4 \\
\hline High-Load & $664(16)$ & $665(18)$ & $683(15)$ \\
RT (ms) & & & 15 \\
\hline \% Error & 16 & 16 & \\
\hline
\end{tabular}




\section{Figure Captions}

Figure 1: Example of the stimulus displays with an emotional distractor present, used in Experiment 1 (Panel A), Experiment 2 (panel B) and Experiment 3 (panel C).

Figure 2: Distractor-interference score on the letter-search task as a function of emotional IAPS pictures and perceptual load in Experiment 1.

Figure 3: Distractor-interference score on the letter-search task as a function of emotional faces and perceptual load in Experiment 2.

Figure 4: Distractor-interference score on the letter-search task as a function of high-value coded distractors and perceptual load in Experiment 3. 

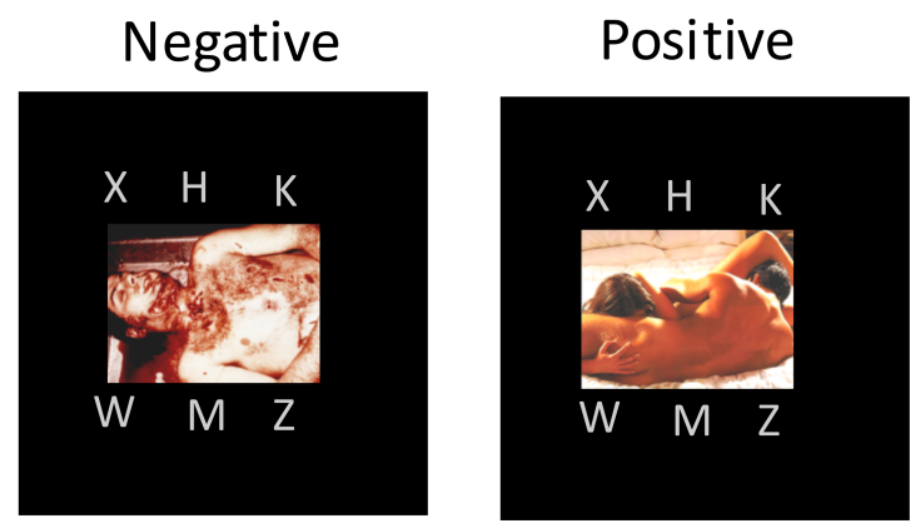

(A)
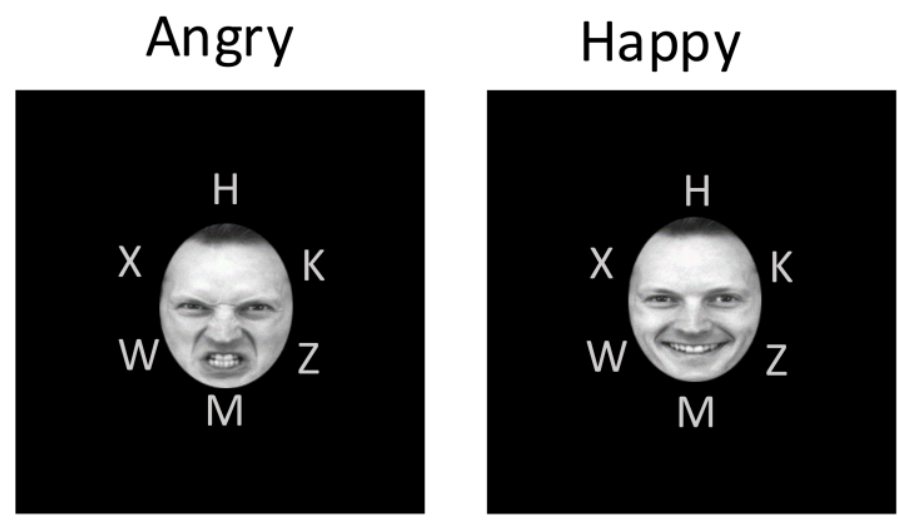

(B)

High-Loss

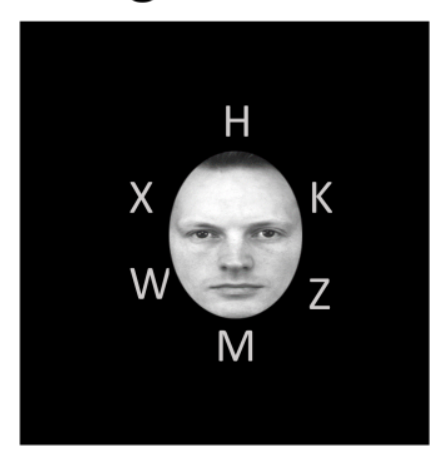

High-Gain

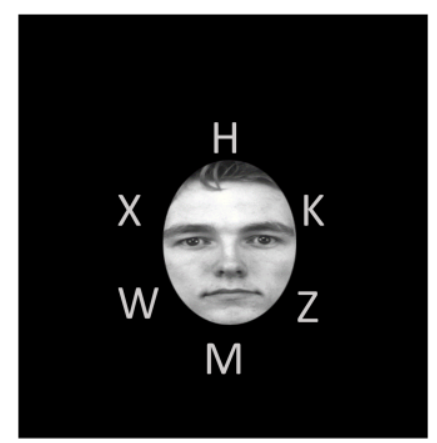

(C)

Figure 1 


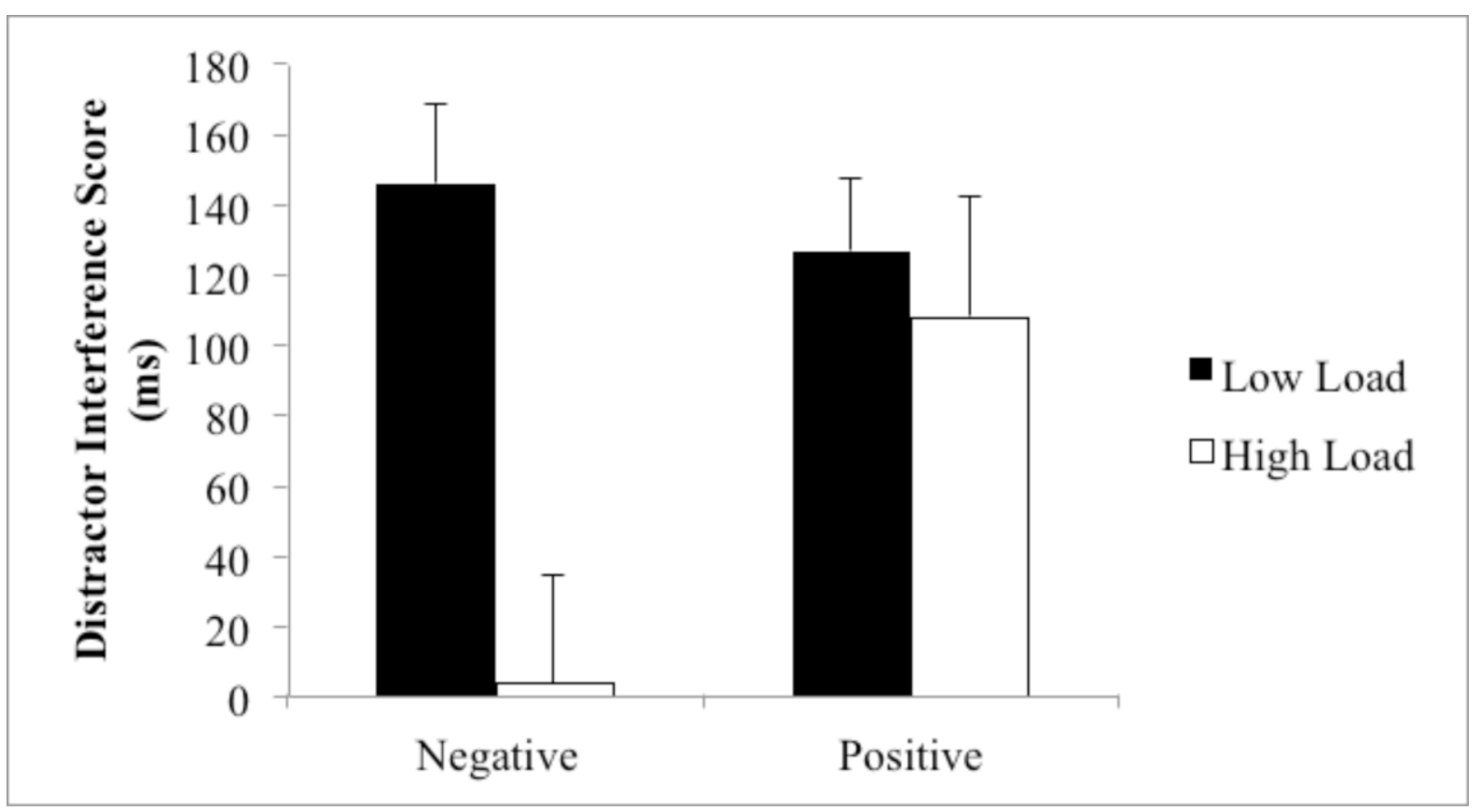

Figure 2 


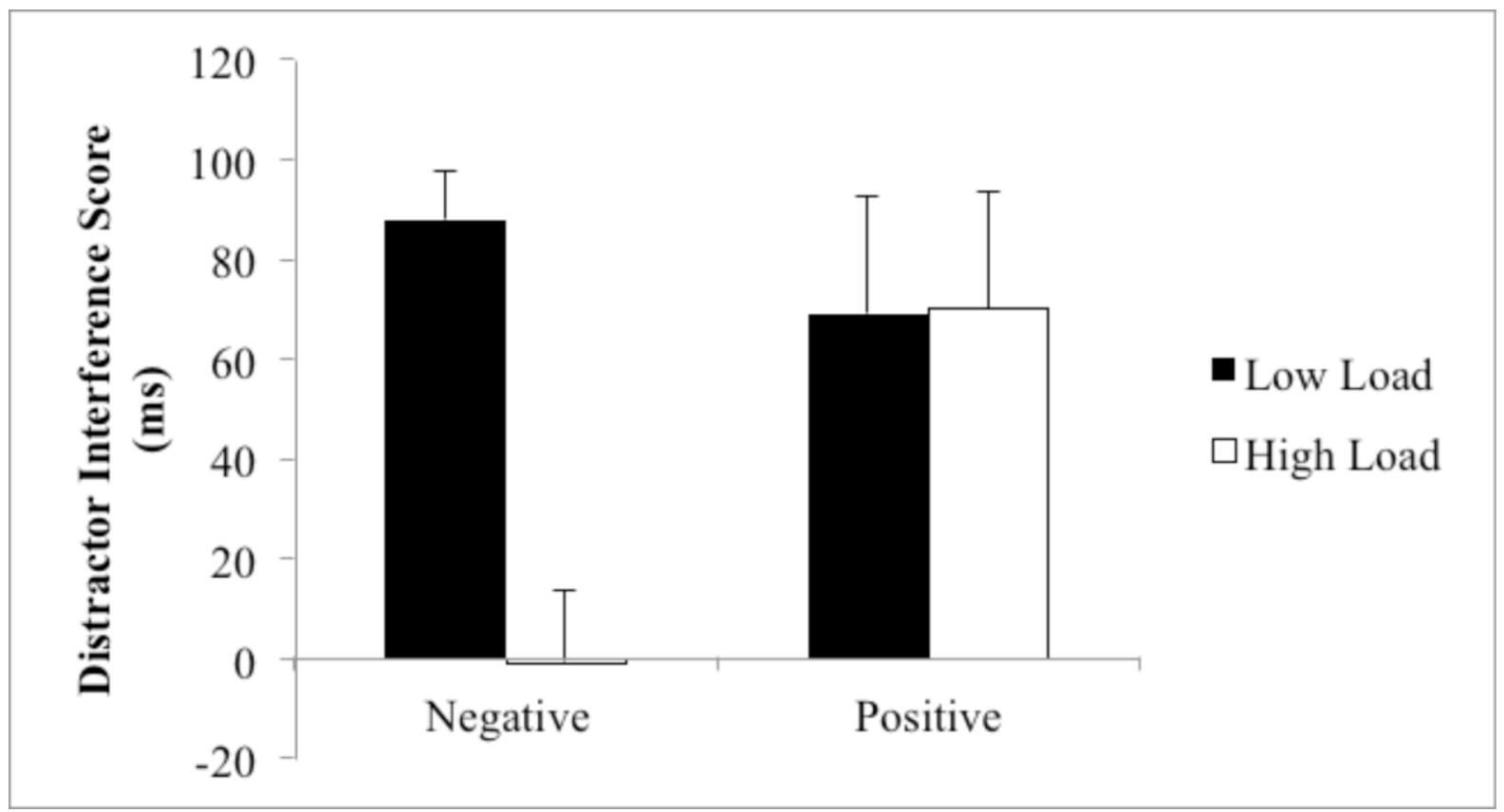

Figure 3 


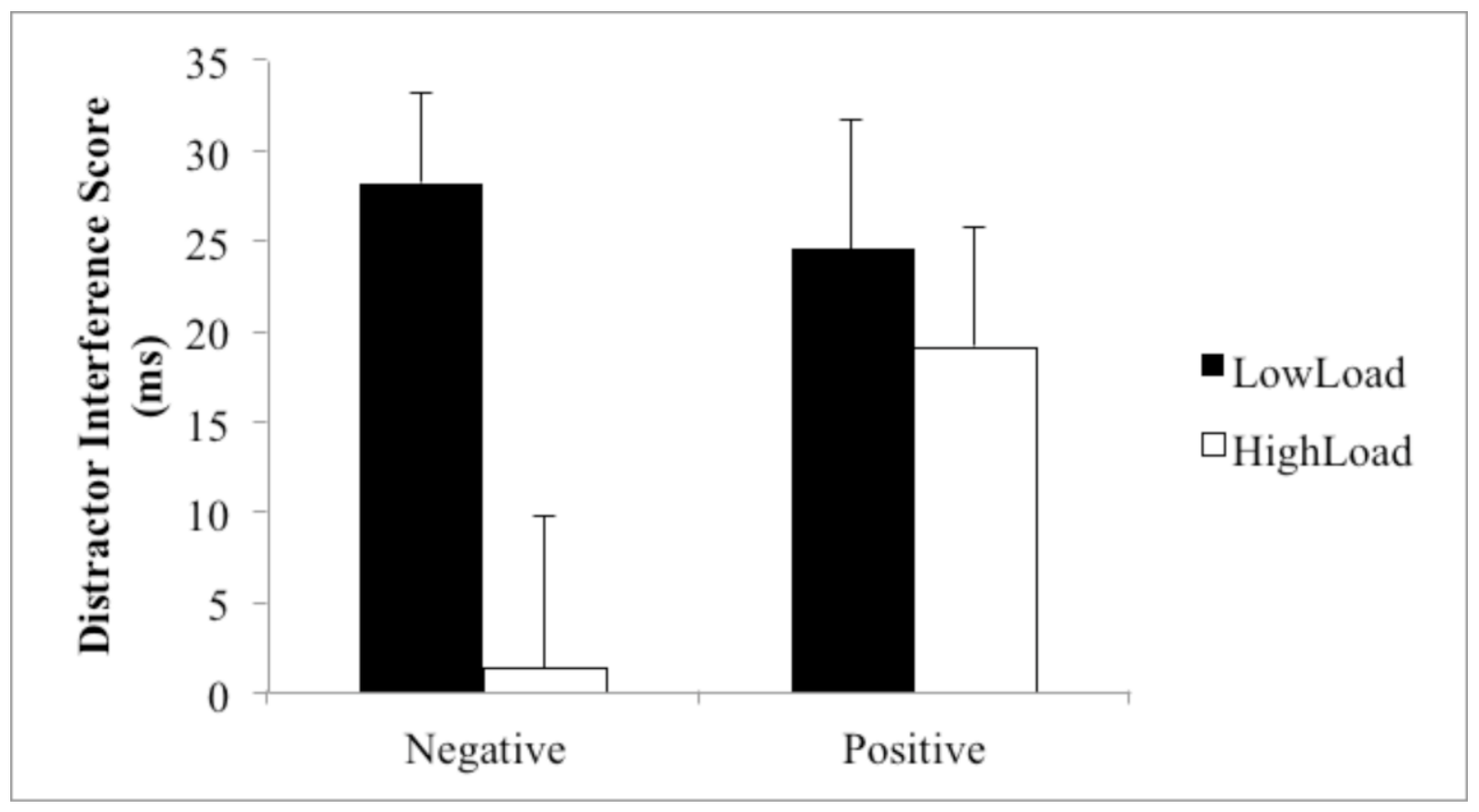

Figure 4 